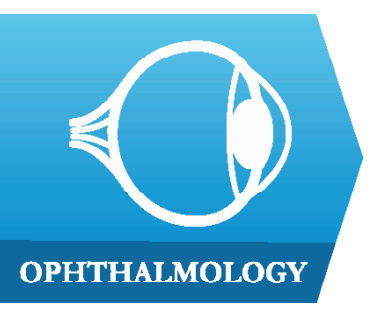

1) Department of Biochemistry and Clinical Biochemistry, Nicolae Testemitanu State University of Medicine and Pharmacy, Republic of Moldova

2) Laboratory of Biochemistry, Nicolae Testemitanu State University of Medicine and Pharmacy, Republic of Moldova

3) Ovisus Medical Private Center, Chisinau, Republic of Moldova

\section{Study of ischemia modified albumin (IMA) as a biomarker in hypertensive retinopathy}

\author{
Ecaterina Pavlovschi ${ }^{1}$, Valeriana Pantea ${ }^{2}$, Djina Borovic ${ }^{3}$, Olga Tagadiuc $^{1}$
}

\begin{abstract}
Background. Hypertension (HTN) is one of the leading causes of morbidity and mortality worldwide. A prompt diagnosis and treatment of hypertensive retinopathy (HR), the leading complication of HTN is pivotal for a better visual outcome. Increased blood pressure on its own cannot fully clarify the development of retinal alterations, therefore an additional pathogenetic mechanism, such as oxidative stress, might be inquired.

The aim of the study was to evaluate the changes in the level of ischemia modified albumin (IMA) in the serum and tears of HR patients in order to establish the predictive value of IMA for the HR progression.

Methods. Serum and tear samples for the measurement of IMA were collected from 90 patients detected primarily with HR, who were not taking any antihypertensive or other drug that could influence the results of the study, divided according to the KeithWagener classification into GI -36 patients with HR grade I, GII - 35 with HR grade II and GIII - 19 with HR grade III. Serum and tear IMA levels were assessed using the $\mathrm{Co}^{2+}$ binding method and expressed as median and interquartile range. KruskalWallis and Mann-Whitney nonparametric tests were used to compare the groups and the Spearman correlation coefficient was calculated (SPSS 23.0), with $\mathrm{p}<0.05$ being statistically significant.
\end{abstract}

Results. The groups showed a statistically significant difference in serum IMA $(\mathrm{p}=0.006)$, the values increasing in parallel with the progression of HR. The serum IMA level in GII increased compared to GI $(+3 \% ; 239.06 \mu \mathrm{M} / \mathrm{L}$ (IQR 75.58) vs 231.77 $\mu \mathrm{M} / \mathrm{L}$ (IQR 104.09), $\mathrm{p}=1.00)$, as well as in GIII patients compared to GII $(+17 \% ; 277.67$ $\mu \mathrm{M} / \mathrm{L}$ (IQR 88.72) vs $239.06 \mu \mathrm{M} / \mathrm{L}$ (IQR 75.58), $\mathrm{p}=0.04$ ). There were no differences in IMA content $(\mathrm{p}=0.160)$, between groups in the tears. No correlations were found between serum and tear IMA levels $(\mathrm{p}=0.134)$, but serum IMA showed a significant moderate strength, positive correlation with the degree of $\mathrm{HR}(\mathrm{r}=0.307, \mathrm{p}=0.003)$.

Conclusion. A progressive enhancement in serum IMA level as HR advanced was identified. Thereby, the results suggest the potential relevance of serum IMA as a sensitive and early biomarker useful for grading and optimal treatment of the patients with HR.

Keywords: ischemia modified albumin, biomarker, oxidative stress, hypertensive retinopathy

\section{Introduction}

Systemic arterial hypertension (HTN) is one of the leading modifiable risk factors of morbidity and mortality worldwide and is related with enhanced risk of cardiovascular, renal, cerebrovascular and retinal diseases. WHO estimates that worldwide approximately $40 \%$ of the persons over
25 years old are hypertensive [1].

The retinal vessels are damaged due to elevated uncontrolled blood pressure, condition known as hypertensive retinopathy (HR) [1]. Less than half of the people with HTN are aware of their status, and many others are aware but not treated, inadequately treated or noncompliant to treatment, although successful treatment 
of HTN reduces the risk of HR [2]. The danger lies also in the fact that the person does not perceive the presence of the disease, due to the lack of prodrome symptoms, that usually develop in the late stages. Also, it must be mentioned that HR acts as a predictor of systemic morbidity and mortality due to target-organ damage [3].

The duration and severity of HTN are directly proportional to the incidence of HR, which ranges from $66.3 \%$ to $83.6 \%$ out of the total hypertensive patients $[2,4]$. The prevalence of HR is higher in Afro-Caribbean as compared to Europeans and higher in women than in men [5].

The uniqueness of the retinal vessels is due to their easy accessibility for a physical examination. It is a well-known fact in the ophthalmology clinic that an asymptomatic hypertensive patient can frequently be first diagnosed with HR and only then referred to the primary care unit to a general practitioner. Physical consultation depends on the dexterity of the ophthalmologist, being somehow subjective, thus the biochemical markers are needed as an additional help in the evaluation of HR stage and for a subsequent correct therapeutic approach.

It is supposed that there are other underlying causes, which play an important role in the development of HR, besides essential and secondary HTN. The researchers associated the clinical manifestations of HR with biochemical markers, such as of inflammation, endothelial cell activation, oxidative stress, angiogenesis, adiponectin, leptin, low birth weight, body mass index and alcohol consumption [4-11].

Low-grade systemic inflammation with its marker - high-sensitivity C-reactive protein (hs-CRP) - was proven to be involved in the pathogenesis of HR [11]. Karaca et al. showed that endothelial dysfunction (as reflected by high levels of von Willebrand factor [12]), which is a mechanism known to be involved in vascular lesions, may promote the development of HR. Researchers found an association of HR with increased plasma leptin level, considered to be a marker of vascular endothelium damage [8]. Persistent microalbuminuria and decreased creatinine clearance in patients with renal dysfunction, were also shown to be a marker for HR and end-organ damage as heart, eye, kidney etc. [10].

Many causes are assigned in order to explain the metabolic and structural changes associated with HR development. The elevation of blood pressure by itself does not fully reflect the extent of retinopathy, thus hypothetically involving other pathogenic mechanisms, such as oxidative stress (OS) [6]. The newest studies suggest that OS, known to be involved in vascular lesions, may promote the development of HR. To explain the pathogenetic role of OS in HR, so far only two markers of OS have been studied: serum gamma-glutamyl transferase (GGT) and serum ferritin levels. Both of them showed a significant increase of the levels in parallel with the development of HR and also showed a positive correlation with the degree of HR [6,7].

IMA is a nonspecific marker of tissue ischemia and of the OS induced by ischemia/reperfusion [13]. During the ischemia/reperfusion, the ROS formation is increased and subsequently, the $\mathrm{N}$-terminal amino acids of IMA are modified. This change decreases the affinity of plasma albumin to bind to heavy metal ions such as cobalt, nickel etc $[14,15]$. Thus, IMA is considered to be a useful biomarker for ischemia/reperfusion.

IMA was first identified in the early 1990s and since then it has been studied in patients with myocardial ischemia [16]. Recent research has found that IMA is an ideal and highly potential biomarker for ischemia/ reperfusion. IMA was mostly studied so far in different cardiac and cerebrovascular diseases associated with ischemia/reperfusion [17-19].

There are few data in literature regarding IMA assessment and role in the retinal diseases, namely in HR.

The aim of the study was to evaluate the changes in the level of IMA in the serum and tears of HR patients in order to establish its predictive value of HR progression.

\section{Material and methods}

\section{Study and selection criteria}

A total of 90 hypertensive patients from the Republic of Moldova, admitted to the Ovisus Medical Center, Chisinau, Republic of Moldova, for a vision check and firstly diagnosed with HR were recruited in our study. The enrolment period was between February 2018 and December 2019.

The diagnosis of HR was confirmed after a detailed specific ophthalmological consult: determination of visual acuity, autorefracto-keratometry, perimetry, anterior and fundus biomicroscopy, ultrasonography, tonometry, gonioscopy, optical coherence tomography (OCT) of the macular area and the papilla of the optic nerve when needed, pachymetry. The classification of HR was based on Keith-Wagener-Barker (KWB) grading system.

The exclusion criteria were: the use of medication that can influence the results of the study, the presence of the metabolic disorders (diabetes, obesity), renal and neurological pathologies, ocular and craniocerebral trauma in the background, optic nerve atrophies of different causes and also of the ocular associated diseases: glaucoma, diabetic retinopathy, acute and chronic inflammatory processes, uveitis.

\section{Sample collection}

Venous blood samples $(5 \mathrm{ml})$ were collected and centrifuged for further testing using the spin speed of 3500 rpm for ten minutes, with further separation of the serum. Tear samples were collected from the tear lake inside the lateral conjunctival sac of the inferior fornix with 
microcapillary tubes. Serum and tears were dispensed into Eppendorf microtubes and frozen $\left(-40^{\circ} \mathrm{C}\right)$ until analysis.

\section{Biochemical analysis}

IMA levels were determined in serum and tear samples, according to the modified cobalt-binding assay described by Gudumac V. and Tagadiuc O., based on the property of proteins to bind $\mathrm{Co}^{2+}$ ions, which causes a decrease in the $\mathrm{Co}^{2+}$ solution color intensity, measured photometrically at $492 \mathrm{~nm}$ [20].

The results were expressed in $\mu \mathrm{M} / \mathrm{L}$, for both serum and tear.

\section{Statistical analysis}

The data were processed using SPSS 23.0 Software. Descriptive statistical methods were used in order to calculate the median and interquartile range (IQR). Kolmogorov-Smirnov and Shapiro-Wilk normality tests were used to analyze data distribution. The homogeneity of variance was determined by Levene's test. To determine if there were significant differences in biomarker levels between the different groups, the non-parametric KruskalWallis test for multiple groups and Mann-Whitney for binary comparisons was applied. Correlation analysis was done using Spearman correlation test. A $p<0.05$ was considered statistically significant.

\section{Ethical issues}

The study was approved by the Research Ethics Committee of Nicolae Testemitanu State University of Medicine and Pharmacy (12.02.2018). All procedures performed in studies involving human participants were in accordance with the ethical standards of the institutional research committee and with the 1964 Helsinki declaration and its later amendments or comparable ethical standards. An informed consent was obtained from all the patients.

\section{Results}

Of the 90 patients included in the study 38 (42.2\%) were male and $52(57.8 \%)$ were female, with a mean age of $59.79 \pm 12.29$ years (range: $38-88$ ).

Patients were divided in 3 groups, considering the degree of HR, according to Keith-Wagner-Barker classification [2]. Group I - 36 patients with $\mathrm{I}^{\text {st }}$ grade of HR; Group II -35 patients with $2^{\text {nd }}$ degree of HR and Group III -19 patients with $3^{\text {rd }}$ degree of HR.

A statistically significant difference in serum IMA $\left(p=0.006^{* *}\right)$ between groups was identified, the values increasing with the progression of HR (Table I).

In paired group comparisons, the serum IMA level in GII increased compared to GI $(+3 \% ; 239.06 \mu \mathrm{M} / \mathrm{L}$ (IQR 75.58 ) vs $231.77 \mu \mathrm{M} / \mathrm{L}$ (IQR 104.09), $\mathrm{p}=1.00$ ), as well as in GIII patients compared to GII $(+17 \% ; 277.67 \mu \mathrm{M} / \mathrm{L}$ (IQR 88.72 ) vs $239.06 \mu \mathrm{M} / \mathrm{L}$ (IQR 75.58), $\mathrm{p}=0.04 *$ ). Statistically significant differences were observed between Groups I and III $(231.77 \mu \mathrm{M} / \mathrm{L}$ (IQR 104.09) vs. $277.67 \mu \mathrm{M} / \mathrm{L}$ (IQR 88.72), $\left.\mathrm{p}=0.005^{* *}\right)$ (Figure 1).
Table I. IMA levels in serum and tears per grade of HR.

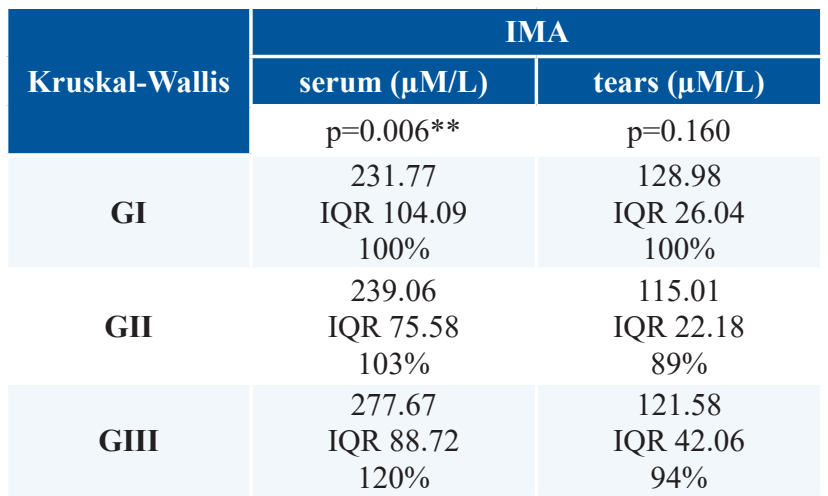

Legend: differences between groups and $\mathrm{p}$ value $<$ : $*<0.05$; $* *<0.01$

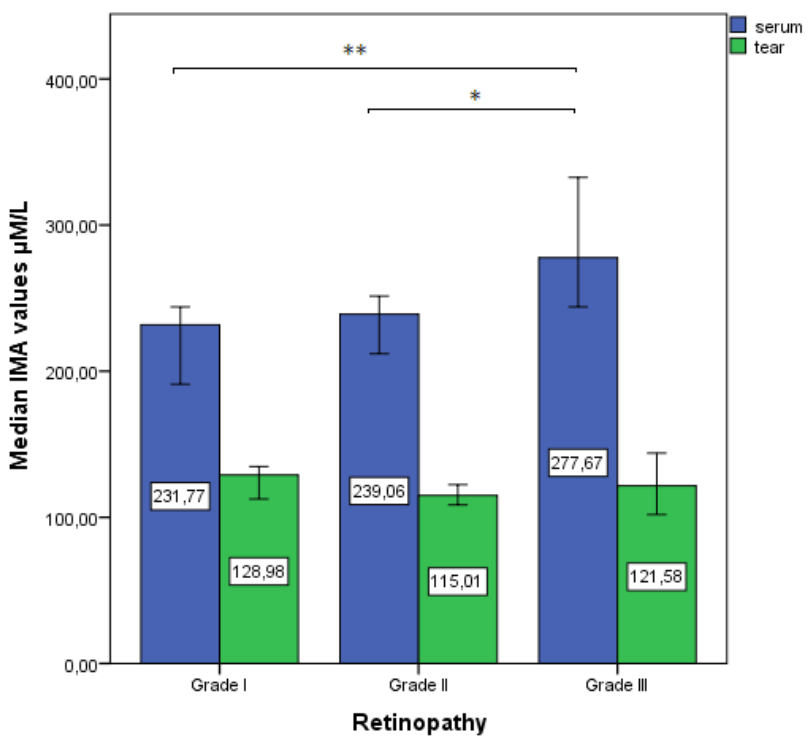

Figure 1. Serum and tear levels of IMA in patients with different grade of HR.

In the tear samples the IMA values were 128.98 $\mu \mathrm{M} / \mathrm{L}$ (IQR 26.04) in the $\mathrm{I}^{\text {st }}$ group, $115.01 \mu \mathrm{M} / \mathrm{L}$ (IQR 22.18 ) in the $\mathrm{II}^{\text {nd }}$ group and $121.58 \mu \mathrm{M} / \mathrm{L}$ (IQR 42.06) in the III ${ }^{\text {rd }}$ group of patients with HR. There were no differences in IMA content $(\mathrm{p}>0.05)$, between groups in the tear samples.

No correlations were found between serum and tear IMA levels ( $\mathrm{r}=0.159, \mathrm{p}=0.134)$, while serum IMA showed a significant medium strength, positive correlation with the degree of HR $\left(\mathrm{r}=0.307^{* *}, \mathrm{p}=0.003\right)$ (Table II). 
Table II. Correlation between the IMA levels in serum and tear and grade of HR.

\begin{tabular}{|c|c|c|c|}
\hline & & IMA level in serum & IMA level in tear \\
\hline \multirow{2}{*}{ Retinopathy } & Correlation coefficient & $0.307 * *$ & -0.126 \\
\hline & Statistical significance, 2-tailed (p) & 0.003 & 0.238 \\
\hline
\end{tabular}

Legend: differences between groups and $\mathrm{p}$ value: $* *<0.01$.

\section{Discussion}

Recent studies have reported a strong association of IMA with ischemic stroke, intracranial hemorrhage, skeletal muscle ischemia, mesenteric ischemia, peripheral atherosclerosis, hyperlipidemia, obesity, metabolic syndrome, hepatosteatosis, preeclampsia, ovarian torsion, fetal distress, $\beta$-thalassemia, diabetes, advanced renal disease and liver cirrhosis [21-26]. Serum IMA value was significantly increased in almost all the above-named cases, due to the role of the OS in the development of these diseases.

OS is incriminated to participate in the development of HTN [26-28]. Experimental evidence shows that ROSs play a pivotal pathological role in the pathogenesis of HTN due to $\mathrm{O}_{2}^{--}$excess and diminished bioavailability of the nitric oxide in the vasculature and kidneys and to ROS-mediated cardiovascular remodeling. Under normal conditions, the ROSs production is controlled and they are kept at low concentration. They function as signaling molecules that maintain the integrity of the vessels, due to their involvement in the regulation of endothelial function and vascular contraction-relaxation balance. Under pathological conditions, enhanced activity of the ROS leads to endothelial dysfunction, an essential pathological mechanism in the development of HTN. Additionally, amplified ROS levels promote vascular smooth muscular cells growth, enhanced contractility, invasion of the monocytes, lipid peroxidation, inflammation and increased deposition of extracellular matrix proteins, all of which are significant factors in hypertensive vascular damage $[26,29,30]$. In HTN, OS systemic biomarkers levels are increased.

Several recent studies show the implication of oxidative stress in the development of HR. So far, only two markers of oxidative stress have been studied. Karaca et al. [6] reported a high level of gamma-glutamyl transferase (GGT) in HR. GGT, an enzyme with a central role in glutathione homeostasis, is important in maintaining adequate concentrations of intracellular glutathione to protect cells against oxidants. Increased serum GGT level is a sensitive marker of oxidative stress [6]. Another study of Coban et al. suppose that there is a relationship between HR and ferritin level, which may be associated with an increased level of oxidative stress [7].

In OS the formation of IMA is determined by the exposure to free radicals, as a result of changes that arise in the microvascular network, including the retinal one. Despite the fact that the mechanisms by which OS occurs and contributes to the development of hypertensive complications are still uncertain, the resulting biochemical modifications contribute to structural and functional changes in the microvasculature. These impairments generate changes in the blood flow of the retina, deterioration of blood-retina barrier and in the end capillary occlusion and ischemia. A continuous reduction of the capillary perfusion will lead to the formation of all well-known signs of HR as cotton-wool spots, intraretinal microvascular abnormalities, venous beading and neovascularization. In ischemic conditions, like in HR described above, the capacity of albumin for binding transition metals such as nickel, copper and cobalt is diminished, thus resulting in the formation of IMA - a different variant of albumin [31-34].

It was shown that HTN is also accompanied with rise in IMA levels [26-28]. Our results are quite similar to those presented. But, to the best of our knowledge, our study showed for the first time the increased levels of serum IMA in HR. The research demonstrated that hypertensive patients with HR have increased IMA levels in the serum, which are positively correlated with the degree of HR $(\mathrm{r}=0.326, \mathrm{p}=0.002)$.

The presence of elevated serum IMA levels in HR and the correlation of the amount of serum IMA with the severity of HR suggest that oxidative stress may be incriminated in the mechanism of HR development. Given the fact that tear secretion is not proportionally affected by the biochemical changes in the blood of hypertensive patients, the lack of correlation between serum IMA and tear IMA does not diminish the importance of serum IMA as a potential biomarker, but it is better to be interpreted in a clinical context.

The self-regulation of the retinal circulation collapses as blood pressure (BP) enhances beyond the critical limit. Moreover, increased BP by itself does not entirely explain the degree of HR [8,9,31,32]. It was attested that regardless of the persistence of elevated blood pressure, retinopathy was resolved [35]. In our study, despite the fact that the BP levels in all three groups were similar, the IMA levels were higher in group 2 than in group 1 and in group 3 than in group 2. Thus, the presence of increased IMA levels in HR and the correlation of the IMA amount with the severity of HR point to the fact that IMA can be used as a marker for the monitoring of HR progression. 


\section{Study limitations}

The study has some limitations. It was conducted on patients that were not taking any antihypertensive treatment or any other treatment that could affect the results, so the results cannot be extended to the patients under any kind of treatment.

IMA in our research is an isolated marker, whose changes cannot be entirely explained either by HTN or HR by themselves, or by the association of both of them. Also, our results cannot answer the question if the high IMA levels precede retinopathy or inversely are a consequence of it. There has not been a logical demonstration that all the retinal signs that appear during development of HR have an independent predictive value, so IMA can be used as an additional marker for risk evaluation and, perhaps for the optimization of the therapeutic approach. Future studies will be helpful in confirming and developing these findings.

\section{Conclusions}

HR it is a recognized manifestation of target organ damage in hypertensive patients. The main intent of screening for HR is that retinal vessels are the sole blood vessels visible on routine examination. The consequences of chronically increased HTN are efficiently visible in the eye as HR and choroidopathy, and this reflects the vascular changes occurring in other systems, but the accuracy of the ophthalmological examination is operator-dependent. Moreover, the ophthalmological evaluation cannot be expressed in measurable indicators and new markers will considerably ease the diagnostic and ensure a better grading of the patients in groups.

Increased serum IMA levels in HR and the correlation of the amount of serum IMA with the severity of HR, depicted in our study, suggests that it could be used as a marker of HR progression and as a predictor of major retinal damage. In addition, it could be used for screening patients with HTN at risk of developing HR.

Further studies are needed to establish the cutoff values of IMA serum levels for stratification of patients according to HR groups in order to optimize the therapeutic plan for preventing the HR progression.

\section{References}

1. Oparil S, Acelajado MC, Bakris GL, Berlowitz DR, Cífková $\mathrm{R}$, Dominiczak AF, et al. Hypertension. Nat Rev Dis Primers 2018;4:18014.

2. Modi P, Arsiwalla T. Hypertensive Retinopathy. [Updated 2020 Apr 27]. In: StatPearls. Treasure Island (FL): StatPearls Publishing; 2020. Available from: https://www.ncbi.nlm.nih. gov/books/NBK525980/

3. Kabedi NN, Mwanza JC, Lepira FB, Kayembe TK, Kayembe DL. Hypertensive retinopathy and its association with cardiovascular, renal and cerebrovascular morbidity in Congolese patients. Cardiovasc J Afr. 2014;25:228-232.
4. Erden S, Bicakci E. Hypertensive retinopathy: incidence, risk factors, and comorbidities. Clin Exp Hypertens. 2012;34:397-401.

5. Chatterjee S, Chattopadhyay S, Hope-Ross M, Lip PL. Hypertension and the eye: changing perspectives. J Hum Hypertens. 2002;16:667-675.

6. Karaca M, Coban E, Felek R, Unal M. The association of oxidative stress with hypertensive retinopathy. Clin Exp Hypertens. 2013;35:16-19.

7. Coban E, Alkan E, Altuntas S, Akar Y. Serum ferritin levels correlate with hypertensive retinopathy. Med Sci Monit. 2010;16:CR92-CR95.

8. Uckaya G, Ozata M, Sonmez A, Kinalp C, Eyileten T, Bingol $\mathrm{N}$, et al. Is leptin associated with hypertensive retinopathy? $\mathrm{J}$ Clin Endocrinol Metab. 2000;85:683-687.

9. Yilmaz MI, Sonmez A, Kilic S, Celik T, Bingol N, Pinar $\mathrm{M}$, et al. The association of plasma adiponectin levels with hypertensive retinopathy. Eur J Endocrinol. 2005;152:233240.

10. Biesenbach G, Zazgornik J. High prevalence of hypertensive retinopathy and coronary heart disease in hypertensive patients with persistent microalbuminuria under short intensive antihypertensive therapy. Clin Nephrol. 1994;41:211-218.

11. Coban E, Nizam I, Topal C, Akar Y. The association of lowgrade systemic inflammation with hypertensive retinopathy. Clin Exp Hypertens. 2010;32:528-531.

12. Karaca M, Coban E, Ozdem S, Unal M, Salim O, Yucel $\mathrm{O}$. The association between endothelial dysfunction and hypertensive retinopathy in essential hypertension. Med Sci Monit. 2014;20:78-82.

13. Żurawska-Płaksej E, Grzebyk E, Marciniak D, SzymańskaChabowska A, Piwowar A. Oxidatively modified forms of albumin in patients with risk factors of metabolic syndrome. J Endocrinol Invest. 2014;37:819-827.

14. Bhakthavatsala Reddy C, Cyriac C, Desle HB. Role of "Ischemia Modified Albumin" (IMA) in acute coronary syndromes. Indian Heart J. 2014;66:656-662.

15. Menon B, Ramalingam K, Krishna V. Study of Ischemia Modified Albumin as a Biomarker in Acute Ischaemic Stroke. Ann Neurosci. 2018;25:187-190.

16. Prakash D. Target organ damage in newly detected hypertensive patients. J Family Med Prim Care. 2019;8:20422046.

17. Bhakthavatsala Reddy C, Cyriac C, Desle HB. Role of "Ischemia Modified Albumin" (IMA) in acute coronary syndromes. Indian Heart J. 2014;66:656-662.

18. Yildirim A, Yildirim S, Topaloglu N, Tekin M, Kucuk A, Erdem $\mathrm{H}$, et al. Correlation of ischemia-modified albumin levels and histopathologic findings in experimental ovarian torsion. Turk J Emerg Med. 2016;16:8-11.

19. Pan D, Li D. Role of ischemia-modified albumin in patients with acute decompensated heart failure. Anatol J Cardiol. 2015;15:618-619.

20. Gudumac V, Tagadiuc O. Method for determining the capacity ischemia modified albumin to bind cobalt. Patent of invention no. MD 4054 of 11.06.2009. Available from: 
http://www.db.agepi.md/Inventions/details/a\%202009\%20 0116/Des a\%202009\%200116.

21. Adly AAM, ElSherif NHK, Ismail EAR, Ibrahim YA, Niazi $\mathrm{G}$, Elmetwally SH. Ischemia-modified albumin as a marker of vascular dysfunction and subclinical atherosclerosis in $\beta$-thalassemia major. Redox Rep. 2017;22:430-438.

22. Piva SJ, Duarte MM, Da Cruz IB, Coelho AC, Moreira AP, Tonello R, et al. Ischemia-modified albumin as an oxidative stress biomarker in obesity. Clin Biochem. 2011;44:345-347.

23. Oran I, Oran B. Ischemia-Modified Albumin as a Marker of Acute Coronary Syndrome: The Case for Revising the Concept of "N-Terminal Modification" to "Fatty Acid Occupation" of Albumin. Dis Markers. 2017;2017:5692583.

24. Timercan T, Timercan V. Assessment of ischemia modified albumin serum level in iso-proterenol-induced myocardial infarction. Biological Markers in Fundamental and Clinical Medicine. 2017;1:4-6.

25. Lazăr C, Vozian M, Pantea V, Mișina A, Tagadiuc O. Ischemia modified albumin in experimental ovarian torsion with and without controlled reperfusion. Rev Romana Med Lab. 2019;27(1):43-50.

26. Kumar A. Prognostic implications of ischemia modified albumin in known cases of 86 elderly hypertensive South Asian aged 56-64 years - a hospital based study. Asian Pacific J Trop Dis. 2014;4:S429-S434.

27. Reddy VS, Pasupuleti P, Perugu B. Implications of ischemia modified albumin levels in hypertension. Asian Pacific J
Trop Dis. 2015;5:S190-S191.

28. Rodrigo R, Libuy M, Feliú F, Hasson D. Oxidative stressrelated biomarkers in essential hypertension and ischemiareperfusion myocardial damage. Dis Markers. 2013;35:773790.

29. Touyz RM. Reactive oxygen species in vascular biology: role in arterial hypertension. Expert Rev Cardiovasc Ther. 2003;1:91-106.

30. Redón J, Oliva MR, Tormos C, Giner V, Chaves J, Iradi A, et al. Antioxidant activities and oxidative stress byproducts in human hypertension. Hypertension. 2013;41:1096-1101.

31. DellaCroce JT, Vitale AT. Hypertension and the eye. Curr Opin Ophthalmol. 2008;19:493-498.

32. Wong TY, Mitchell P. The eye in hypertension. Lancet. 2007;369:425-435.

33. Reddy VS, Sethi S, Agrawal P, Gupta N, Garg R. Ischemia modified albumin (IMA) and albumin adjusted-IMA (AAIMA) as biomarkers for diabetic retinopathy. Nepal J Ophthalmol. 2016;7:117-123.

34. Turk A, Nuhoglu I, Mentese A, Karahan SC, Erdol H, Erem C. The relationship between diabetic retinopathy and serum levels of ischemia-modified albumin and malondialdehyde. Retina. 2011;31:602-608.

35. Lowenthal MN, Zimlichman R. Resolution of hypertensive retinopathy despite persistent high diastolic pressure. South Med J. 1993;86:190-193. 